\begin{tabular}{|c|l|}
\hline Title & Singularities of improper affine spheres and surfaces of constant Gaussian curvature \\
\hline Author(s) & Ishikawa, Go-o; Machida, Y oshinori \\
\hline Citation & $\begin{array}{l}\text { International journal of mathematics, 17(3), 269-293 } \\
\text { https://doi.org/L0.1142/S0129167X 06003485 }\end{array}$ \\
\hline Issue Date & 2006-03 \\
\hline Doc URL & http://hdl.handle.net/2115/8512 \\
\hline Rights & copyright(c)2006 for W orld Scientific \\
\hline Type & article (author version) \\
\hline File Information & 石川IGI-YM-IJM.pdf \\
\hline
\end{tabular}

Instructions for use 


\title{
Singularities of improper affine spheres and surfaces of constant Gaussian curvature
}

\author{
Go-o ISHIKAWA* and Yoshinori MACHIDA ${ }^{\dagger}$
}

\begin{abstract}
We study the equation for improper (parabolic) affine spheres from the view point of contact geometry and provide the generic classification of singularities appearing in geometric solutions to the equation as well as their duals. We also show the results for surfaces of constant Gaussian curvature and for developable surfaces. In particular we confirm that generic singularities appearing in such a surface are just cuspidal edges and swallowtails.
\end{abstract}

Dedicated to Professor Tohru Morimoto on his 60th birthday

\section{Introduction.}

Let $f(x, y)$ be a $C^{\infty}$ function on $\mathbf{R}^{2}$ satisfying the unimodular Hessian equation:

$$
\operatorname{Hess}(f)=\left|\begin{array}{cc}
\frac{\partial^{2} f}{\partial x^{2}} & \frac{\partial^{2} f}{\partial x \partial y} \\
\frac{\partial^{2} f}{\partial y \partial x} & \frac{\partial^{2} f}{\partial y^{2}}
\end{array}\right|= \pm 1 .
$$

Then the graph $z=f(x, y)$ in $\mathbf{R}^{3}$ is an improper affine sphere with the affine normal vector field $\partial / \partial z[\mathrm{~N}-\mathrm{S}]$. In this note we study the equations "Hessian $= \pm 1$ " and singularities of improper affine spheres. Also we study the equations of constant Gaussian curvature $K=c$ for surfaces in $\mathbf{R}^{3}$. We provide the results on singularities of geometric solutions to $K=-1$ ("pseudo-spherical surfaces"), $K=1$ ("sphere-like surfaces") and $K=0$ ("developable surfaces").

The importance of the study on singularities comes from the well-known classical results: A smooth global solution in $\mathbf{R}^{3}$ to Hess $=1$ is the graph of a quadratic polynomial function (Jörgens' theorem $[\mathrm{Jr}][\mathrm{C}][\mathrm{Po}]$, cf. Bernstein's theorem $[\mathrm{B} 2][\mathrm{N}])$. Therefore other solutions necessarily have singularities. Besides we know: A compact surface in $\mathbf{R}^{3}$ with constant positive Gaussian curvature is a sphere (Liebmann's theorem $[\mathrm{O}]$ ). Therefore compact solution surfaces to $K=1$ other than spheres have necessarily singularities. Moreover, there are no complete surface in $\mathbf{R}^{3}$ with constant negative Gaussian curvature (Hilbert's theorem[Hi], cf. [Ts]). Therefore solution surfaces to the equation $K=-1$ have necessarily singularities.

As well-known, the equations Hess $=c$ and $K=c$ are regarded as Monge-Ampère equations, and they have been studied from both geometric and analytic aspects. Note that, for a surface $z=f(x, y)$, we have $K=\frac{f_{x x} f_{y y}-f_{x y}^{2}}{\left(1+f_{x}^{2}+f_{y}^{2}\right)^{2}}$ and the equation $K=c$ turns to be

$$
f_{x x} f_{y y}-f_{x y}^{2}=c\left(1+f_{x}^{2}+f_{y}^{2}\right)^{2} .
$$

2000 Mathematics Subject Classification: Primary. 53C42, 53A15, Secondary. 58K40, 58A15.

Keywords: Monge-Ampère equation, Lagrangian pair, geometric solution, cuspidal edge, swallowtail, open umbrella.

*Partially supported by Grants-in-Aid for Scientific Research, No. 14340020.

†Partially supported by Grants-in-Aid for Scientific Research, (C) No. 14540097. 
Then we observe that there are associated to these equations an additional geometric structure, the structure of Lagrangian pair ([I-Ma]), and based on that, we proceed to detailed study on the singularities of solutions beyond usually expected.

Consider the equation $f_{x x} f_{y y}-f_{x y}^{2}=c$ for a surface $z=f(x, y)$ in $x y z$-space $\mathbf{R}^{3}$. Geometrically the equation can be written into the differential system

$$
\omega=c d x \wedge d y-d p \wedge d q=0, \quad \theta=d z-p d x-q d y=0
$$

on $x y z p q$-space $\mathbf{R}^{5}, p, q$ representing $z_{x}, z_{y}$ respectively. Then we have the contact distribution $D=\{\theta=0\}$ with the symplectic structure $\left.d \theta\right|_{D}$ in the tangent bundle $T \mathbf{R}^{5}$. Moreover $E_{1}=$ $\left\{v \in D \mid i_{v}(d x \wedge d y)=0\right\}$ and $E_{2}=\left\{v \in D \mid i_{v}(d p \wedge d q)=0\right\}$ are Lagrangian subbundles of $D$, where $i_{v}$ denotes the interior product by $v$. In fact $E_{1}$ (resp. $E_{2}$ ) is generated by $\frac{\partial}{\partial p}, \frac{\partial}{\partial q}$ (resp. by $\left.\frac{\partial}{\partial x}+p \frac{\partial}{\partial z}, \frac{\partial}{\partial y}+q \frac{\partial}{\partial z}\right)$. Then the double Legendrian fibration

$$
\begin{aligned}
& M=\mathbf{R}^{5} \\
& \pi_{1} \swarrow \quad \searrow \quad \pi_{2} \\
& W_{1}=\mathbf{R}^{3} \quad W_{2}=\mathbf{R}^{3}
\end{aligned}
$$

is induced. The first projection $\pi_{1}$ is defined by $(x, y, z, p, q) \mapsto(x, y, z)$ and the second projection $\pi_{2}$ is defined by $(x, y, z, p, q) \mapsto(p, q, p x+q y-z)$.

The differential system associated to the equation $K=c$ in the Euclidean 3 -space $\mathbf{R}^{3}$ is defined on the unit tangent bundle $\mathbf{R}^{3} \times S^{2}$ of $\mathbf{R}^{3}$ by the 2 -form

$$
\omega=c\left(y_{1} d x_{2} \wedge d x_{3}+y_{2} d x_{3} \wedge d x_{1}+y_{3} d x_{1} \wedge d x_{2}\right)-\left(y_{1} d y_{2} \wedge d y_{3}+y_{2} d y_{3} \wedge d y_{1}+y_{3} d y_{1} \wedge d y_{2}\right),
$$

and the contact form $\theta=y_{1} d x_{1}+y_{2} d x_{2}+y_{3} d x_{3}$. Here $\left(x_{1}, x_{2}, x_{3} ; y_{1}, y_{2}, y_{3}\right)$ is the system of coordinates on $\mathbf{R}^{3} \times \mathbf{R}^{3}$ restricted to $\mathbf{R}^{3} \times S^{2}$. We set $D=\{\theta=0\} \subset T\left(\mathbf{R}^{3} \times S^{2}\right)$, and two Lagrangian subbundles of $D$ :

$$
\begin{aligned}
E_{1} & =\left\{v \in D \mid i_{v}\left(y_{1} d x_{2} \wedge d x_{3}+y_{2} d x_{3} \wedge d x_{1}+y_{3} d x_{1} \wedge d x_{2}\right)=0\right\} \\
& =\left\{v=\eta_{1} \frac{\partial}{\partial y_{1}}+\eta_{2} \frac{\partial}{\partial y_{2}}+\eta_{3} \frac{\partial}{\partial y_{3}} \mid v \text { is tangent to } S^{2}\right\}, \\
E_{2} & =\left\{v \in D \mid i_{v}\left(y_{1} d y_{2} \wedge d y_{3}+y_{2} d y_{3} \wedge d y_{1}+y_{3} d y_{1} \wedge d y_{2}\right)=0\right\} \\
& =\left\{v=\xi_{1} \frac{\partial}{\partial x_{1}}+\xi_{2} \frac{\partial}{\partial x_{2}}+\xi_{3} \frac{\partial}{\partial x_{3}} \mid \xi_{1} y_{1}+\xi_{2} y_{2}+\xi_{3} y_{3}=0\right\} .
\end{aligned}
$$

Then we have the double Legendrian fibration

$$
\begin{gathered}
M=\mathbf{R}^{3} \times S^{2} \\
\pi_{1} \swarrow \\
W_{1}=\mathbf{R}^{3} \\
\searrow \quad \pi_{2}=\mathbf{R} \times S^{2},
\end{gathered}
$$

where $\pi_{1}(x, y)=x, \pi_{2}(x, y)=(x \cdot y, y)$ for $(x, y) \in \mathbf{R}^{3} \times S^{2} \subset \mathbf{R}^{3} \times \mathbf{R}^{3}$, and $x \cdot y$ is the inner product (the height function).

In general, let $M$ be a contact manifold of dimension $2 n+1$ with a contact structure $D \subset$ $T M$. A Monge-Ampère system on $M$ is an exterior differential system $\mathcal{M}$ generated locally by 
a contact form $\theta$ for $D$ and an $n$-form $\omega$ on $M$. The geometric formulation of Monge-Ampère systems, originally due to T. Morimoto [M1][M2] and V.V. Lychagin [L], naturally and intrinsically generalizes the classical Monge-Ampère equations, and describes several fundamental equations in geometry, analysis and physics. As explained above, we have Monge-Ampère systems associated to the equations Hessian $=c$ and $K=c$ respectively.

An immersion $f: N^{n} \rightarrow M$ from a manifold $N$ of dimension $n$ is called a Legendrian immersion if $f_{*}(T N) \subset D(\subset T M)$, where $f_{*}: T N \rightarrow T M$ is the differential of $f$. A Legendrian immersion $f: N \rightarrow M$ is called a geometric solution of a Monge-Ampère system $\mathcal{M}$ if $f^{*} \mathcal{M}=0$. If $\mathcal{M}$ is given by a contact form $\theta$ and an $n$-form $\omega$, then the condition reads $f^{*} \theta=0, f^{*} \omega=0$.

We lift any solution surface $z=f(x, y)$ to Hess $=c$ uniquely to a geometric solution to Hess $=c$ in $\mathbf{R}^{5}$, by setting $p=f_{x}, q=f_{y}$. Then the $\pi_{2}$-projection of the lifting is nothing but the affine dual of the original surface. Also we lift any surface in $\mathbf{R}^{3}$ with $K=c$ with a fixed co-orientation, uniquely to a geometric solution to $K=c$ in $\mathbf{R}^{3} \times S^{2}$, by using the Gauss map (unit normals). The $\pi_{2}$-projection of the lifting is the pedal surface in $\mathbf{R} \times S^{2} \cong \mathbf{R}^{3} \backslash\{0\}$ of the original surface. To describe singularities of solution surfaces, we start to study geometric solutions and then the both $\pi_{1}$ and $\pi_{2}$-projections of them.

In this paper we show the following result:

Theorem 1.1 A generic geometric solution to Hess $=1$ (resp. to Hess $=-1, K=1$, or $K=-1$ ) has only cuspidal edges and swallowtails as singularities. More strictly, any geometric solution $N^{2} \rightarrow M^{5}=\mathbf{R}^{5}$ or $\mathbf{R}^{3} \times S^{2}$ to Hess $=1$ (resp. to Hess $=-1, K=1$, or $K=-1$ ) can be locally approximated near each point in $N$ in $C^{\infty}$ topology by a geometric solution $f: U \rightarrow M$ such that, for any $x_{0} \in U$, one of the following assertions (i), (ii), (iii), (iv) holds, with respect to the Legendrian fibrations $\pi_{1}: M \rightarrow W_{1}=\mathbf{R}^{3}$ and $\pi_{2}: M \rightarrow W_{2}=\mathbf{R}^{3}$ or $\mathbf{R} \times S^{2}$ :

(i) $\pi_{1} \circ f:\left(U, x_{0}\right) \rightarrow W_{1}$ is an immersion at $x_{0}$, and $\pi_{2} \circ f:\left(U, x_{0}\right) \rightarrow W_{2}$ is an immersion at $x_{0}$.

(ii) $\pi_{1} \circ f$ has the cuspidal edge at $x_{0}$, and $\pi_{2} \circ f$ has the cuspidal edge at $x_{0}$.

(iii) $\pi_{1} \circ f$ has the swallowtail at $x_{0}$, and $\pi_{2} \circ f$ has the cuspidal edge at $x_{0}$.

(iv) $\pi_{1} \circ f$ has the cuspidal edge at $x_{0}$, and $\pi_{2} \circ f$ has the swallowtail at $x_{0}$.

The significance of Theorem 1.1 is twofold.

First, Theorem 1.1 is a collection of four theorems: Four results are independent to each other, since four equations have different properties to each other and we need to analyze geometric solutions for each equations. Neverthless we get the same list of generic singularities as a result.

Note also that the equation Hess $=c(c>0)($ resp. Hess $=c(c<0))$ is equivalent to Hess $=1$ (resp. Hess $=-1$ ), by the contactomorphism $(x, y, z, p, q) \mapsto\left(\sqrt{|c|} x, \sqrt{|c|} y, z, \frac{1}{\sqrt{|c|}} p, \frac{1}{\sqrt{|c|}} q\right)$. Similarly the equation $K=c(c>0)$ (resp. $K=c(c<0)$ ) is equivalent to $K=1$ (resp. $K=-1$ ) by the contactomorphism $(x, y) \rightarrow(\sqrt{|c|} x, y)$.

Second, the classification result of Theorem 1.1 differs from that for generic Legendrian submanifolds:

Proposition 1.2 For a generic Legendrian immersion $N^{2} \rightarrow \mathbf{R}^{5}$ (not necessarily a geometric solution), one of the following holds:

(a) $\pi_{1} \circ f$ is an immersion, and $\pi_{2} \circ f$ is an immersion at $x_{0}$.

(b) $\pi_{1} \circ f$ is the cuspidal edge, and $\pi_{2} \circ f$ is an immersion at $x_{0}$.

(c) $\pi_{1} \circ f$ is an immersion, and $\pi_{2} \circ f$ is the cuspidal edge at $x_{0}$.

(d) $\pi_{1} \circ f$ is the cuspidal edge, and $\pi_{2} \circ f$ is the cuspidal edge at $x_{0}$.

(e) $\pi_{1} \circ f$ is the swallowtail, and $\pi_{2} \circ f$ is an immersion at $x_{0}$.

(f) $\pi_{1} \circ f$ is an immersion and $\pi_{2} \circ f$ is the swallowtail at $x_{0}$. 
Note that Proposition 1.2 is a straightforward result from ordinary Legendrian singularity theory $([\mathrm{A}-\mathrm{G}-\mathrm{V}][\mathrm{Z}])$. In fact the result is described by the generic combination of two stratifications on the plane by $A_{3}, A_{2}, A_{1}$-singularities via two Legendrian fibrations $\pi_{1}$ and $\pi_{2}$ respectively.

Contrary to Proposition 1.2, we observe the simultaneous occurrence of singularities of $\pi_{1}$ and $\pi_{2}$-projections generically, in Theorem 1.1. For example, the property of the equation Hess $=1$ affects it. In fact from the equations $\omega=d x \wedge d y-d p \wedge d q=0, \theta=d z-p d x-q d y=0$, we see $\pi_{1} \circ f$ is immersive at $x_{0}$ if and only if $\pi_{2} \circ f$ is immersive at $x_{0}$, therefore $\pi_{1} \circ f$ is singular at $x_{0}$ if and only if $\pi_{2} \circ f$ is singular at $x_{0}$.

Remark 1.3 In [K-R-S-U-Y], it is shown that the generic singularities on flat surfaces in the hyperbolic 3-space $H^{3}$ are cuspidal edges and swallowtails, based on the representation formula obtained in [K-U-Y]. Also a useful criterion on singularities is established in [K-R-S-U-Y], so called "KRSUY" criterion. To show Theorem 1.1, we analyze by means of power series expansions and transversality arguments, in each case Hess $=1$, Hess $=-1, K=1, K=-1$ and Hess $=0(K=0)$ on $\mathbf{R}^{3}$. To finish up the classification in each case, we apply the "KRSUY" criterion.

For the improper affine spheres, the global complex representation is given in [F-M-M]. Moreover the notion of improper affine maps is introduced in [Mar] in connection with special Lagrangian immersions, and improper affine spheres with singularities are considered. Furthermore the classification of singular improper affine spheres can be reduced to the classification of singular flat fronts in $H^{3}$ as shown in [K-R-S-U-Y]. This is communicated to the first author by M. Umehara and K. Yamada. Note that Theorem 1.1 gives the classification of singularities not only for improper affine spheres, but also for their affine duals as well.

It seems to be natural to conjecture that the generic singularities on surfaces of constant negative Gaussian curvature should be cuspidal edges and swallowtails. (See [Mc] for the pictures of singularities appearing on surfaces of constant negative curvature). Moreover, by numerical experiments, it can be conjectured that also the generic singularities on surfaces of constant positive Gaussian curvature should be cuspidal edges and swallowtails([Kob]). Theorem 1.1 answers the conjectures affirmatively.

Remark 1.4 Theorems 1.1 describes generic singularities for both $\pi_{1}$ and $\pi_{2}$-projections. Note that it is most natural to classify geometric solutions under the equivalence preserving the structure of double fibration, namely, by posing that the contactomorphism on $M$ should be taken in common for $\pi_{1}$ and $\pi_{2}$. However, then it is hopeless to expect a finite list of classification as in Theorem 1.1 , since such classification has functional moduli in general.

Now recall the fundamental notions appeared in Theorem 1.1.

Let $\pi:\left(\mathbf{R}^{2 n+1}, 0\right) \rightarrow\left(\mathbf{R}^{n+1}, 0\right)$ be a Legendrian fibration. Two Legendrian immersions $f, g:\left(\mathbf{R}^{n}, 0\right) \rightarrow\left(\mathbf{R}^{2 n+1}, 0\right)$ are called Legendre equivalent if there exist a contactomorphism $\Phi:\left(\mathbf{R}^{2 n+1}, 0\right) \rightarrow\left(\mathbf{R}^{2 n+1}, 0\right)$, a diffeomorphism $\sigma:\left(\mathbf{R}^{n}, 0\right) \rightarrow\left(\mathbf{R}^{n}, 0\right)$ and a diffeomorphism $\varphi:\left(\mathbf{R}^{n+1}, 0\right) \rightarrow\left(\mathbf{R}^{n+1}, 0\right)$ such that the following diagram commutes:

$$
\begin{array}{ccccc}
\left(\mathbf{R}^{n}, 0\right) & \stackrel{f}{\longrightarrow} & \left(\mathbf{R}^{2 n+1}, 0\right) & \stackrel{\pi}{\longrightarrow} & \left(\mathbf{R}^{n+1}, 0\right) \\
\sigma \downarrow & & \Phi \downarrow & & \varphi \downarrow \\
\left(\mathbf{R}^{n}, 0\right) & \stackrel{g}{\longrightarrow} & \left(\mathbf{R}^{2 n+1}, 0\right) & \stackrel{\pi}{\longrightarrow} & \left(\mathbf{R}^{n+1}, 0\right) .
\end{array}
$$

Let $\pi:\left(\mathbf{R}^{5}, 0\right) \rightarrow \mathbf{R}^{3}$ be a germ of Legendrian fibration with respect to the contact form $\theta=d z-p d x-q d y$. A Legendrian immersion $f:\left(\mathbf{R}^{2}, 0\right) \rightarrow\left(\mathbf{R}^{5}, 0\right)$ is called a cuspidal edge (or $A_{2}$ briefly) with respect to $\pi$, if $f$ is Legendre equivalent to

$$
(x, y, z, p, q)=\left(u, v^{2}, \frac{2}{3} v^{3}, 0, v\right) .
$$

In this case we say that $\pi \circ f$ has the cuspidal edge at 0 . A Legendrian immersion $f:\left(\mathbf{R}^{2}, 0\right) \rightarrow$ $\left(\mathbf{R}^{5}, 0\right)$ is called a swallowtail (or $A_{3}$ briefly) with respect to $\pi$, if $f$ is Legendre equivalent to

$$
(x, y, z, p, q)=\left(u, v^{3}+u v, \frac{3}{4} v^{4}+\frac{1}{2} u v^{2},-\frac{1}{2} v^{2}, v\right) .
$$


In this case we say that $\pi \circ f$ has the swallowtail at 0 .

The immersion can be called " $A_{1}$-singularity".

Example 1.5 Let $f:\left(\mathbf{R}^{2}, 0\right) \rightarrow \mathbf{R}^{5}$ be a map-germ defined by

$$
\begin{aligned}
x & =u \\
y & =2 u v+3 u^{2} v-v^{3} \\
z & =\frac{1}{3} u^{3}+u v^{2}+\frac{1}{4} u^{4}+\frac{3}{2} u^{2} v^{2}-\frac{3}{4} v^{4} \\
p & =u^{2}-v^{2}+u^{3}-3 u v^{2} \\
q & =v .
\end{aligned}
$$

Then $f$ is a geometric solution to Hess $=1$. Moreover $\pi_{1} \circ f=(x, y, z)$ has the swallowtail at 0 and $\pi_{2} \circ f=(x, y, p x+q y-z)$ has the cuspidal edge at 0 . See Figure 1 . Note that

$$
\tilde{z}=p x+q y-z=\frac{2}{3} u^{3}+\frac{3}{4} u^{4}-\frac{3}{2} u^{2} v^{2}-\frac{1}{4} v^{4} .
$$
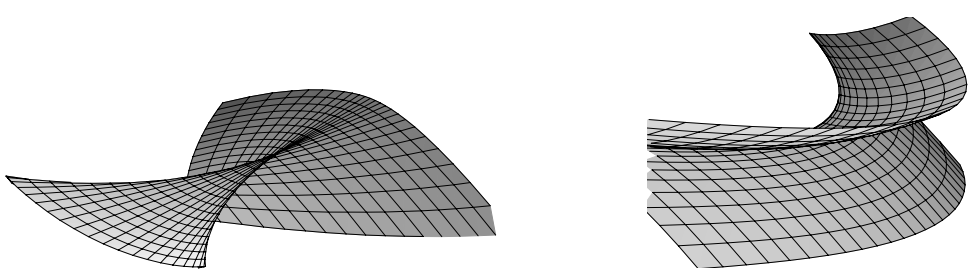

Figure 1: An improper affine sphere with the swallowtail singularity (left) and with the cuspidal edge singularity (right) in its dual.

The solution surfaces to the equation Hess $=0$ or $K=0$, in the case $c=0$, are so called "developable surfaces" [Is]. Then we face a different situation with respect to dual surfaces from the cases $c \neq 0$. Actually we have the following classification result:

Theorem 1.6 A generic geometric solution to Hess $=0$ (resp. $K=0$ ) has only cuspidal edges $\left(A_{2}\right)$ and swallowtails $\left(A_{3}\right)$ as singularities with respect to $\pi_{1}$, while it collapses to a generic immersed space curve by $\pi_{2}$.

Note that the same classification result for generic singularities of developable surfaces has been given in [Iz], with respect to the topology on the space of tangent developables to space curves.

Also we have a result on the geometric solutions which are not necessarily immersions; generalized geometric solutions. We call a $C^{\infty}$ mapping $f: N^{n} \rightarrow M^{2 n+1}$, which is not necessarily an immersion, an integral mapping if $f_{*}(T N) \subset D(\subset T M)$. If $D=\{\theta=0\}$, then the condition means that $f^{*} \theta=0$. An integral mapping is called a generalized geometric solution to a Monge-Ampère system $\mathcal{M}$ generated by a contact form $\theta$ and an $n$-form $\omega$ if $f^{*} \theta=0, f^{*} \omega=0$.

An integral map-germ $f:\left(\mathbf{R}^{2}, 0\right) \rightarrow M^{5}$ is called an open umbrella if $f$ is contactomorphic to

$$
(u, v) \mapsto(x, y, z, p, q)=\left(u, v^{2}, u v^{3}, v^{3}, \frac{3}{2} u v\right),
$$

by a contactomorphism not necessarily preserving the Legendre fibrations. The open umbrella appears as the Legendre lifting of the tangent developable surface to a space curve where the 
curvature does not vanish and the torsion simply vanishes ([Is]). Therefore the open umbrella is a generalized geometric solution to Hess $=0$ (resp. $K=0)([\mathrm{I}-\mathrm{Mo}])$.

Then we show the following:

Proposition 1.7 An open umbrella cannot be a generalized geometric solution to Hess $=c(c \neq 0)$ (resp. $K=c(c \neq 0))$.

In the next section, we recall the criterion of cuspidal edges $\left(A_{2}\right)$ and swallowtails $\left(A_{3}\right)$ established in [K-R-S-U-Y]. In $\S 3$, we deal with geometric solutions to the equations Hess $= \pm 1$, in connection with the classical solutions to the Laplace equation and to the wave equation, and show Theorems 1.1 for them. In $\S 4$, we treat geometric solutions to the equations $K= \pm 1$, by means of the method of Cauchy-Kovalevskaya's type to complete the proof of Theorem 1.1. In $\S 5$, we give the proofs of Theorem 1.6 and Proposition 1.7.

\section{A criterion for cuspidal edges and swallowtails.}

In the recent paper [K-R-S-U-Y], a simple criterion is established for cuspidal edge singularities and swallowtails singularities of wave fronts. The criterion is very easy to handle, and we are going to use them effectively for our classification results in the following sections. We modify the criterion slightly according to the situation to which we are going to apply.

Let $f:\left(\mathbf{R}^{2},\left(u_{0}, v_{0}\right)\right) \rightarrow \mathbf{R}^{5}$ be a germ of Legendrian immersion,

$$
f(u, v)=(x(u, v), y(u, v), z(u, v), p(u, v), q(u, v)),
$$

for the standard contact form $\theta=d z-p d x-q d y$ on $\mathbf{R}^{5}$ with the Legendrian fibration $\pi: \mathbf{R}^{5} \rightarrow \mathbf{R}^{3}$, $\pi(x, y, z, p, q)=(x, y, z)$. Set $g=\pi \circ f$. Suppose $\left(u_{0}, v_{0}\right)$ is a singular point, namely a nonimmersive point, of $g$. We call the singular point $\left(u_{0}, v_{0}\right)$ non-degenerate if $\Delta=\operatorname{det}\left(\begin{array}{c}x_{u}, x_{v} \\ y_{u}, y_{v}\end{array}\right)$ : $\left(\mathbf{R}^{2},\left(u_{0}, v_{0}\right)\right) \rightarrow(\mathbf{R}, 0)$ is submersive. Note that $g_{u} \times g_{v}=\Delta \cdot(-p,-q, 1)$ so that the singular locus of $g$ is given by $\Delta=0$ on $\left(\mathbf{R}^{2},\left(u_{0}, v_{0}\right)\right)$.

Suppose a singular point $\left(u_{0}, v_{0}\right)$ of $g$ is non-degenerate. Then the singular locus can be parametrized by an immersed curve $\gamma:(\mathbf{R}, 0) \rightarrow\left(\mathbf{R}^{2},\left(u_{0}, v_{0}\right)\right)$. Moreover we see $g$ has rank 1 along $\gamma(t)$ near $t=0$. Then the kernel field of $g_{*}$ is generated by a non-vanishing vector field $\eta:(\mathbf{R}, 0) \rightarrow T \mathbf{R}^{2}$ along $\gamma$ so that $\left(g_{\gamma(t)}\right)_{*}(\eta(t))=0$.

Then the criterion is given as follows:

Proposition 2.1 (Proposition 1.3 of [K-R-S-U-Y]) Let $p$ is a non-degenerate singular point of $g=\pi \circ f:\left(\mathbf{R}^{2},\left(u_{0}, v_{0}\right)\right) \rightarrow \mathbf{R}^{3}$ for a Legendrian immersion $f:\left(\mathbf{R}^{2},\left(u_{0}, v_{0}\right)\right) \rightarrow \mathbf{R}^{5}$. Then $f$ is a cuspidal edge at $\left(u_{0}, v_{0}\right)$ with respect to $\pi$ if and only if $\operatorname{det}\left(\gamma^{\prime}(0), \eta(0)\right) \neq 0$. On the other hand, $f$ is a swallowtail at $\left(u_{0}, v_{0}\right)$ with respect to $\pi$ if and only if $\operatorname{det}\left(\gamma^{\prime}(0), \eta(0)\right)=0$ and $\left.\frac{\partial}{\partial t} \operatorname{det}\left(\gamma^{\prime}(t), \eta(t)\right)\right|_{t=0} \neq 0$.

Example 2.2 Just to make sure, let us check that our normal form $(x, y, z, p, q)=\left(u, v^{2}, \frac{2}{3} v^{3}, 0, v\right)$ of the cuspidal edge satisfies the criterion. In this case, we have $\Delta=2 v$. We can set $\gamma(t)=(t, 0)$ and $\eta(t)=(0,1)$. Therefore $\operatorname{det}\left(\gamma^{\prime}(0), \eta(0)\right)=1$.

For the normal form $(x, y, z, p, q)=\left(u, v^{3}+u v, \frac{3}{4} v^{4}+\frac{1}{2} u v^{2},-\frac{1}{2} v^{2}, v\right)$ of the swallowtail at $\left(u_{0}, v_{0}\right)=(0,0)$, we have $\Delta=3 v^{2}+u$. Then $\eta(t)=(0,1)$. For any immersion $\gamma(t)=(u(t), v(t))$ which parametrizes $\{\Delta=0\}$, we have $3 v(t)^{2}+u(t)=0$, and $u^{\prime}(0)=0$. Moreover, since $v(0)=$ $0, v^{\prime}(0) \neq 0$, we have $\left.\frac{\partial}{\partial t} \operatorname{det}\left(\gamma^{\prime}(t), \eta(t)\right)\right|_{t=0}=u^{\prime \prime}(0)=6 v^{\prime}(0)^{2} \neq 0$. 


\section{Singularities of improper affine spheres and their duals.}

We observe the fact that the ellipticity of the equation Hess $=1$ implies the "rigidity" of solutions, controlled by holomorphic or harmonic functions, while the hyperbolicity of the equation Hess $=-1$ implies the "softness" of solutions, controlled by $C^{\infty}$ functions.

Then the key point of the proof of Theorem 1.1 lies on the fact that even in elliptic cases there exist enough solutions implying the validity of a transversality theorem.

Before starting the detailed analysis, we remark that our objects are homogeneous: Consider the group $G$ of equi-affine transformations preserving the vector field $\frac{\partial}{\partial z}$. Then $G$ acts transitively on $\mathbf{R}^{5}$, on $\mathbf{R}^{3}$, and the dual $\mathbf{R}^{3}$ in the natural way. Moreover $\pi_{1}: \mathbf{R}^{5} \rightarrow \mathbf{R}^{3}$ and $\pi_{2}: \mathbf{R}^{5} \rightarrow \mathbf{R}^{3}$ are $G$-equivariant. Furthermore the differential system $\omega=0, \theta=0$ on $\mathbf{R}^{5}$ is $G$-invariant. Throughout this section, we use the homogeneity for simplifying the calculations.

First, let us consider the equation Hess $=1$ on $\mathbf{R}^{5}$.

Let $f:\left(\mathbf{R}^{2}, 0\right) \rightarrow \mathbf{R}^{5}$ be a germ of a geometric solution to Hess $=1$. We assume $f$ is an immersion, and $f^{*} \theta=0$ for the contact form $\theta=d z-p d x-q d y$ and $f^{*} \omega=0$ for the 2-form $\omega=d x \wedge d y-d p \wedge d q$. Set

$$
f(u, v)=(x(u, v), y(u, v), z(u, v), p(u, v), q(u, v)) .
$$

First we observe the following:

Lemma 3.1 Suppose either $\left(\pi_{1} \circ f\right)_{*}: T_{0} \mathbf{R}^{2} \rightarrow T_{0} \mathbf{R}^{3}$ or $\left(\pi_{2} \circ f\right)_{*}: T_{0} \mathbf{R}^{2} \rightarrow T_{0} \mathbf{R}^{3}$ is injective. Then both $\left(\pi_{1} \circ f\right)_{*}$ and $\left(\pi_{2} \circ f\right)_{*}$ are injective.

Proof: Since $f^{*}(d z-p d x-q d y)=0$, the condition that $\left(\pi_{1} \circ f\right)_{*}: T_{0} \mathbf{R}^{2} \rightarrow T_{0} \mathbf{R}^{3}$ is injective is equivalent to that $\left(\pi_{1} \circ f\right)^{*}(d x \wedge d y) \neq 0$ at 0 . Similarly, since $f^{*}(d(x p+y q-z)-x d p-y d q)=0$, the condition that $\left(\pi_{2} \circ f\right)_{*}: T_{0} \mathbf{R}^{2} \rightarrow T_{0} \mathbf{R}^{3}$ is injective is equivalent to that $\left(\pi_{2} \circ f\right)^{*}(d p \wedge d q) \neq 0$. On the other hand, $0=f^{*}(d x \wedge d y-d p \wedge d q)=\left(\pi_{1} \circ f\right)^{*}(d x \wedge d y)-\left(\pi_{2} \circ f\right)^{*}(d p \wedge d q)$, we see $\left(\pi_{1} \circ f\right)^{*}(d x \wedge d y)=\left(\pi_{2} \circ f\right)^{*}(d p \wedge d q)$. Thus we have the result.

Moreover we have:

Lemma 3.2 Suppose both $\left(\pi_{1} \circ f\right)_{*}$ and $\left(\pi_{2} \circ f\right)_{*}$ are not injective at 0 . Then $\left(\pi_{1} \circ f\right)_{*}$ has rank 1 and $\left(\pi_{2} \circ f\right)_{*}$ has rank 1 at 0 .

Proof: Assume that $\left(\pi_{1} \circ f\right)_{*}$ is not injective and dose not have rank 1. Then $\left(\pi_{1} \circ f\right)_{*}$ has rank 0 , which means that $f_{*}\left(T_{0} \mathbf{R}^{2}\right)$ is contained in $E_{1}$. Since $\left(E_{1}\right)_{f(0)}$ projects to $T_{\left(\pi_{2} \circ f\right)(0)} \mathbf{R}^{3}$ injectively by $\left(\pi_{2}\right)_{*}$, we see $\left(\pi_{2} \circ f\right)_{*}$ must be injective. Thus we see $\left(\pi_{1} \circ f\right)_{*}$ has rank 1 . By the symmetric argument, we have also that $\left(\pi_{2} \circ f\right)_{*}$ has rank 1 .

Furthermore we have:

Lemma 3.3 Suppose both $\left(\pi_{1} \circ f\right)_{*}$ and $\left(\pi_{2} \circ f\right)_{*}$ are not injective at 0 . Then we have $(x \circ f, q \circ f)_{*}$ : $T_{0} \mathbf{R}^{2} \rightarrow T_{0} \mathbf{R}^{2}$ is isomorphic or $(y \circ f, p \circ f)_{*}: T_{0} \mathbf{R}^{2} \rightarrow T_{0} \mathbf{R}^{2}$ is isomorphic.

Proof: By Lemma 3.2, we see $d(x \circ f)(0) \neq 0$ or $d(y \circ f)(0) \neq 0$. Let $d(x \circ f)(0) \neq 0$. Since $f$ is a Legendrian immersion, we see $(x, p),(x, q),(y, p)$ or $(y, q)$ composed with $f$ is a local diffeomorphism. Assume $d(x \circ f)$ and $d(p \circ f)$ are linearly independent at 0 . Since $f^{*}(d \theta)=0$, we see $0 \neq d(p \circ f) \wedge d(x \circ f)=-d(q \circ f) \wedge d(y \circ f)$ at 0 . Therefore $d(q \circ f)(0)$ and $d(y \circ f)(0)$ must be linearly independent. Besides, we are supposing that $d(x \circ f)(0)$ and $d(y \circ f)(0)$ are linearly dependent. Therefore we have that $d(x \circ f)(0)$ and $d(q \circ f)(0)$ are linearly independent. If $d(y \circ f)(0) \neq 0$, then similarly we have $d(y \circ f)(0)$ and $d(p \circ f)(0)$ are linearly independent. 
Now suppose either $\left(\pi_{1} \circ f\right)_{*}: T_{0} \mathbf{R}^{2} \rightarrow T_{0} \mathbf{R}^{3}$ or $\left(\pi_{2} \circ f\right)_{*}: T_{0} \mathbf{R}^{2} \rightarrow T_{0} \mathbf{R}^{3}$ is not injective. By Lemmata 3.1, 3.2, 3.3, we may assume $x=u, q=v$ by a coordinate transformation of $\left(\mathbf{R}^{2}, 0\right)$ and by the isomorphism $(x, y, z, p, q) \mapsto(y, x, z, q, p)$ of the system. Thus we set

$$
f(u, v)=(u, y(u, v), z(u, v), p(u, v), v) .
$$

By the condition $d z=p d u+v d y$, we have that $d p \wedge d u+d v \wedge d y=0$. Then we have

$$
\frac{\partial p}{\partial v}=-\frac{\partial y}{\partial u}
$$

Also by the condition $\omega=d p \wedge d v-d u \wedge d y=0$, we have

$$
\frac{\partial p}{\partial u}=\frac{\partial y}{\partial v} .
$$

Thus we obtain the Cauchy-Riemann equation. Therefore we see the complex valued function $p+\sqrt{-1} y:\left(\mathbf{R}^{2}, 0\right) \rightarrow \mathbf{C}$ is holomorphic with the complex coordinate $u+\sqrt{-1} v$.

Remark 3.4 Consider a contact transformation $L: \mathbf{R}^{5} \rightarrow \mathbf{R}^{5}$ defined by $L(x, y, z, p, q)=(x, q, z-$ $y q, p,-y)$, which is called a partial Legendre transformation. Then $L^{*}(d p \wedge d y-d q \wedge d x)=$ $-(d x \wedge d y-d p \wedge d q)=-\omega$. Therefore, as well known, the equation Hess $=1$ is contactomorphic to the Laplace equation $z_{x x}+z_{y y}=0$. Similarly, we see the equation Hess $=-1$ is contactomorphic to the wave equation $z_{x x}-z_{y y}=0$. Thus the "nonlinear" equation Hess $= \pm 1$, or $r t-s^{2}= \pm 1$ in the classical Goursat's notation, is equivalent to the "linear" equation $r \pm t=0$. Actually we have used this procedure of Legendrian transformation, and naturally we have got the Cauchy-Riemann equation (resp. the wave equation). However note that the Legendrian transformation $L$ does not preserve the structure of the Lagrangian pair, and also that the Cauchy-Riemann equation we have got is not over the $x y$-plane but rather over the $u v$-plane, the parameter plane of the geometric solution.

Thus we have:

Proposition 3.5 Let $f:\left(\mathbf{R}^{2}, 0\right) \rightarrow \mathbf{R}^{5}$ be a germ of a geometric solution of the Monge-Ampère system associated to the equation Hess $=1$. Then there exists a germ of a holomorphic function $h=p+\sqrt{-1} y:\left(\mathbf{R}^{2}, 0\right)=(\mathbf{C}, 0) \rightarrow(\mathbf{C}, 0)$ of $u+\sqrt{-1} v$ such that $f$ is Legendre equivalent to $f_{h}=(u, y(u, v), z(u, v), p(u, v), v)$, up to a diffeomorphism on $\left(\mathbf{R}^{2}, 0\right)$ and a contactomorphism on $\mathbf{R}^{5}$ preserving $\left(\mathcal{M}, E_{1}, E_{2}\right)$, defined by the line integral

$$
z(u, v)=\int_{(0,0)}^{(u, v)}\left(p(u, v)+v \frac{\partial y}{\partial u}\right) d u+v \frac{\partial y}{\partial v} d v .
$$

Example 3.6 Consider the holomorphic function

$$
h=(u+\sqrt{-1} v)^{2}=u^{2}-v^{2}+\sqrt{-1}(2 u v) .
$$

Set $p=u^{2}-v^{2}$ and $y=2 u v$. Then we have $f_{h}=\left(u, 2 u v, \frac{1}{3} u^{3}+u v^{2}, u^{2}-v^{2}, v\right)$. For the function $h=\sqrt{-1}(u+\sqrt{-1} v)^{2}$ we have $f_{h}=\left(u, u^{2}-v^{2},-\frac{2}{3} v^{3},-2 u v, v\right)$.

More generally, for a holomorphic function

$$
h=\left(a_{1}+\sqrt{-1} b_{1}\right)(u+\sqrt{-1} v)+\left(a_{2}+\sqrt{-1} b_{2}\right)(u+\sqrt{-1} v)^{2}+\left(a_{3}+\sqrt{-1} b_{3}\right)(u+\sqrt{-1} v)^{3}+\cdots,
$$


we have $f_{h}=(x, y, z, p, q)$ with

$$
\begin{aligned}
x= & u \\
y= & a_{1} v+b_{1} u+a_{2}(2 u v)+b_{2}\left(u^{2}-v^{2}\right)+a_{3}\left(3 u^{2} v-v^{3}\right)+b_{3}\left(u^{3}-3 u v^{2}\right)+\cdots \\
z= & a_{1}\left(\frac{1}{2} u^{2}+\frac{1}{2} v^{2}\right)+a_{2}\left(\frac{1}{3} u^{3}+u v^{2}\right)+b_{2}\left(-\frac{2}{3} v^{3}\right) \\
& +a_{3}\left(\frac{1}{4} u^{4}+\frac{3}{2} u^{2} v^{2}-\frac{3}{4} v^{4}\right)+b_{3}\left(-2 u v^{3}\right)+\cdots \\
p= & a_{1} u-b_{1} v+a_{2}\left(u^{2}-v^{2}\right)+b_{2}(-2 u v)+a_{3}\left(u^{3}-3 u v^{2}\right)+b_{3}\left(-3 u^{2} v+v^{3}\right)+\cdots \\
q= & v .
\end{aligned}
$$

This example parametrizes all geometric solutions to Hess $=1$ with projection singularities, up to the equivalence and up to their 3-jets. Namely, for any geometric solution, its germ at each point can be represented as above for some coordinates $u, v$ centered at the point.

To be precise, the argument goes as follows: Let $U$ be an open subset in the $u v$-plane $\mathbf{R}^{2}$. We identify the 3-jet space $J^{3}\left(U, \mathbf{R}^{3}\right)$ with the submanifold of $J^{3}\left(U, \mathbf{R}^{5}\right)$ consisting of 3-jets $j^{3} f\left(x_{0}\right)$ with a form

$$
f(u, v)=(u, y(u, v), z(u, v), p(u, v), v) .
$$

Moreover we identify $J^{3}(2,3)$ with the submanifold of $J^{3}(2,5)=\left\{j^{3} g(0) \mid g:\left(\mathbf{R}^{2}, 0\right) \rightarrow\left(\mathbf{R}^{5}, 0\right)\right\}$ consisting of 3-jets $j^{3} g(0)$ with $x \circ g=u$ and $q \circ g=v$. Consider the submersion

$$
\Phi: J^{3}\left(U, \mathbf{R}^{5}\right) \rightarrow J^{3}(2,5)
$$

by $\Phi\left(j^{3} f\left(u_{0}, v_{0}\right)\right)=j^{3}(\bar{f}(0))$, where $\bar{f}:\left(\mathbf{R}^{3}, 0\right) \rightarrow\left(\mathbf{R}^{5}, 0\right)$ is defined by $\bar{f}(u, v)=f\left(u+u_{0}, v+\right.$ $\left.v_{0}\right)-f\left(u_{0}, v_{0}\right)$. Then $\Phi$ maps $J^{3}\left(U, \mathbf{R}^{3}\right)$ onto $J^{3}(2,3)$. So, the submersion

$$
\Phi: J^{3}\left(U, \mathbf{R}^{3}\right) \rightarrow J^{3}(2,3)
$$

is induced. Let $\mathcal{G}=\mathcal{G}_{U}$ be the 3 -jet space of geometric solutions $\left(U,\left(u_{0}, v_{0}\right)\right) \rightarrow \mathbf{R}^{5}$ with a form

$$
f(u, v)=(u, y(u, v), z(u, v), p(u, v), v) .
$$

Moreover we identify $\mathbf{R}^{6}$ with the submanifold in $J^{3}(2,3)$ consisting of $j^{3}\left(f_{h}\right)(0)$ described as above. Then we have seen $\Phi^{-1}\left(\mathbf{R}^{6}\right)=\mathcal{G}$. Thus we see $\mathcal{G}$ is a manifold of dimension 11 and $\Phi: \mathcal{G} \rightarrow \mathbf{R}^{6}$ is a submersion.

Now the proof of Theorem 1.1 for the equation Hess $=1$ is achieved as follows: Consider the family of 3 -jets of geometric solutions of the above form parametrized by parameters $a_{1}, a_{2}, a_{3}, b_{1}, b_{2}, b_{3}$.

The singular locus of $\pi_{1} \circ f$ is described by

$$
\Delta=y_{v}=a_{1}+2 a_{2} u-2 b_{2} v+3 a_{3}\left(u^{2}-v^{2}\right)-6 b_{3} u v+\cdots=0,
$$

while also the singular locus of $\pi_{2} \circ f$ is described by

$$
\Delta=p_{u}=p_{v}=a_{1}+2 a_{2} u-2 b_{2} v+3 a_{3}\left(u^{2}-v^{2}\right)-6 b_{3} u v+\cdots=0 .
$$

Consider the hypersurface $\left\{a_{1}=0\right\}$ in $\mathbf{R}^{6}$. Then $\Phi^{-1}\left(\left\{a_{1}=0\right\}\right) \subset \mathcal{G}$ is also a hypersurface.

Let $f_{h}: U \rightarrow \mathbf{R}^{5}$ be the geometric solution to Hess $=1$ defined by a holomorphic function $h$ : $U\left(\subset \mathbf{R}^{2}=\mathbf{C}\right) \rightarrow \mathbf{C}$. Consider the mapping $\Psi(h): U \rightarrow \mathcal{G}$ defined by $\Psi(h)\left(u_{0}, v_{0}\right)=j^{3} f_{h}\left(u_{0}, v_{0}\right)$. Then, by a small perturbation $\tilde{h}(z)=h(z)+\alpha z+\beta z^{2}+\gamma z^{3}$ by a complex polynomial $(\alpha, \beta, \gamma \in \mathbf{C})$, we can make $\Psi(\tilde{h}): U \rightarrow \mathcal{G}$ transverse to $\Phi^{-1}\left(\left\{a_{1}=0\right\}\right)$. Then the locus $\left\{a_{1}=0\right\}$ is a smooth curve in $U$.

If (i) $a_{1} \neq 0$, then both $\pi_{1} \circ f$ and $\pi_{2} \circ f$ are immersive.

Moreover by a perturbation of $h$ if necessary, we may suppose, along the curve $\left\{a_{1}=0\right\}$, there occurs only three cases: (ii) $a_{1}=0, a_{2} \neq 0, b_{2} \neq 0$, (iii) $a_{1}=0, a_{2} \neq 0, b_{2}=0, a_{3} \neq 0$, or (iv) $a_{1}=0, a_{2}=0, b_{2} \neq 0, a_{3} \neq 0$. 
We apply the criterion Proposition 2.1 to our case to verify the singularity type of $\pi_{1} \circ f$ (resp. $\left.\pi_{2} \circ f\right)$.

The singularities are non-degenerate since $\left(a_{2}, b_{2}\right) \neq(0,0)$.

Now suppose (iii) $a_{1}=0, a_{2} \neq 0, b_{2}=0, a_{3} \neq 0$. Then we have

$$
\begin{aligned}
& x=u \\
& y=b_{1} u+a_{2}(2 u v)+a_{3}\left(3 u^{2} v-v^{3}\right)+b_{3}\left(u^{3}-3 u v^{2}\right)+\cdots \\
& z=a_{2}\left(\frac{1}{3} u^{3}+u v^{2}\right)+a_{3}\left(\frac{1}{4} u^{4}+\frac{3}{2} u^{2} v^{2}-\frac{3}{4} v^{4}\right)+b_{3}\left(-2 u v^{3}\right)+\cdots \\
& p=-b_{1} v+a_{2}\left(u^{2}-v^{2}\right)+a_{3}\left(u^{3}-3 u v^{2}\right)+b_{3}\left(-3 u^{2} v+v^{3}\right)+\cdots \\
& q=v
\end{aligned}
$$

Then we have, for $\left(u_{0}, v_{0}\right)=(0,0)$,

$$
y_{v}=2 a_{2} u+3 a_{3}\left(u^{2}-v^{2}\right)-6 b_{3} u v+\cdots .
$$

Take a parametrization $\gamma(t)=(u(t), v(t))$ of the singular locus $\left\{y_{v}=0\right\}$ of $\pi_{1} \circ f$ satisfying $\gamma(0)=(0,0), \gamma^{\prime}(0) \neq(0,0)$. Then we see $u^{\prime}(0)=0 u^{\prime \prime}(0) \neq 0$ if and only if $a_{3} \neq 0$. Since we can take $\eta(t)=(0,1)$ as a kernel field, we have $\operatorname{det}\left(\gamma^{\prime}(0), \eta(0)\right)=u^{\prime}(0)=0$ and $\left.\frac{\partial}{\partial t} \operatorname{det}\left(\gamma^{\prime}(t), \eta(t)\right)\right|_{t=0}=$ $u^{\prime \prime}(0) \neq 0$. On the other hand, for a parametrization $\gamma(t)=(u(t), v(t))$ of the singular locus $\left\{p_{u}=0\right\}$ of $\pi_{2} \circ f$,

$$
p_{u}=2 a_{2} u+3 a_{3}\left(u^{2}-v^{2}\right)-6 b_{3} u v+\cdots,
$$

we have $u^{\prime}(0)=0$, so $v^{\prime}(0) \neq 0$. Since we can take $\eta(t)=(1,0)$ as a kernel field for $\pi_{2} \circ f$, we have $\operatorname{det}\left(\gamma^{\prime}(0), \eta(0)\right)=v^{\prime}(0) \neq 0$

Therefore, by Proposition 2.1, $\pi_{1} \circ f$ is the swallowtail and $\pi_{2} \circ f$ is the cuspidal edge in the case (iii).

In the case (iv) $a_{1}=0, b_{1}=0, a_{2}=0, b_{2} \neq 0, a_{3} \neq 0$, we see, similarly to the case (iii), $\pi_{1} \circ f$ is the cuspidal edge and $\pi_{2} \circ f$ is the swallowtail. Moreover in the case (ii) $a_{2} \neq 0, b_{2} \neq 0$, we see both $\pi_{1} \circ f$ and $\pi_{2} \circ f$ are the cuspidal edges.

Thus the proof of Theorem 1.1 for the equation Hess $=1$ is completed.

Next we consider the equation Hess $=-1$. In this case we set

$$
\theta=d z-p d x-q d y, \quad \omega=d x \wedge d y+d p \wedge d q .
$$

Let $f:\left(\mathbf{R}^{2}, 0\right) \rightarrow \mathbf{R}^{5}$ be a germ of an geometric solution to $\mathcal{M}=\langle\theta, \omega\rangle$. Then up to equivalence, we can write

$$
f(u, v)=(u, y(u, v), z(u, v), p(u, v), v),
$$

and we have the equation:

$$
\frac{\partial p}{\partial v}=-\frac{\partial y}{\partial u}, \quad \frac{\partial p}{\partial u}=-\frac{\partial y}{\partial v}
$$

For this wave equation, we get

$$
y=\varphi(u+v)+\psi(u-v), \quad p=-\varphi(u+v)+\psi(u-v),
$$

for smooth functions $\varphi, \psi$.

If

$$
\begin{aligned}
& \varphi=\varphi_{1} t+\varphi_{2} t^{2}+\varphi_{3} t^{3}+\cdots, \\
& \psi=\psi_{1} t+\psi_{2} t^{2}+\psi_{3} t^{3}+\cdots,
\end{aligned}
$$

where $\varphi_{i}, \psi_{j}$ are real numbers, then we have the expansions

$$
\begin{aligned}
& y=\varphi_{1}(u+v)+\psi_{1}(u-v)+\varphi_{2}(u+v)^{2}+\psi_{2}(u-v)^{2}+\varphi_{3}(u+v)^{3}+\varphi_{3}(u-v)^{3}+\cdots \\
& p=-\varphi_{1}(u+v)+\psi_{1}(u-v)-\varphi_{2}(u+v)^{2}+\psi_{2}(u-v)^{2}-\varphi_{3}(u+v)^{3}+\varphi_{3}(u-v)^{3}+\cdots,
\end{aligned}
$$


up to 3 -jets. Moreover we have

$$
\begin{aligned}
z= & \int_{(0,0)}^{(u, v)} p d x+q d y \\
= & \frac{1}{2} \varphi_{1}\left(-u^{2}+v^{2}\right)+\frac{1}{2} \psi_{1}\left(u^{2}-v^{2}\right)+\frac{1}{3} \varphi_{2}\left(-u^{3}+3 u v^{2}+2 v^{3}\right)+\frac{1}{3} \psi_{2}\left(u^{3}-3 u v^{2}-2 v^{3}\right) \\
& +\frac{1}{4} \varphi_{3}\left(-u^{4}+6 u^{2} v^{2}+8 u v^{3}+3 v^{3}\right)+\frac{1}{4} \psi_{3}\left(u^{4}-6 u^{2} v^{2}+8 u v^{3}-3 v^{3}\right)+\cdots
\end{aligned}
$$

Then we see Theorem 1.1 holds for the Monge-Ampère system of Hess $=-1$ by the same way.

Remark 3.7 To describe the jets of solutions to Hess $=-1$, we can also expand as a "split holomorphic function"

$$
p-j y=\left(a_{1}+j b_{1}\right)(u+j v)+\left(a_{2}+j b_{2}\right)(u+j v)^{2}+\left(a_{3}+j b_{3}\right)(u+j v)^{3}+\cdots,
$$

using the split complex number $u+j v$, where $j^{2}=1$, not $j^{2}=-1$, and $u, v$ are real numbers, so as

$$
\begin{aligned}
& p=a_{1} u+b_{1} v+a_{2}\left(u^{2}+v^{2}\right)+b_{2}(2 u v)+a_{3}\left(u^{3}+3 u v^{2}\right)+b_{3}\left(3 u^{2} v+v^{3}\right)+\cdots, \\
& y=-a_{1} v-b_{1} u-a_{2}(2 u v)-b_{2}\left(u^{2}+v^{2}\right)-a_{3}\left(3 u^{2} v+v^{3}\right)-b_{3}\left(u^{3}+3 u v^{2}\right)+\cdots .
\end{aligned} .
$$

In fact the coefficients are related by $a_{k}=-\varphi_{k}+\psi_{k}, b_{k}=-\varphi_{k}-\psi_{k}$. Nevertheless we have to remark that the equation Hess $=-1$ admits infinitely flat nonzero perturbations of geometric solutions, while "the theorem of identity" holds for solutions to Hess $=1$.

\section{Singularities of surfaces of constant Gaussian curvature and their duals.}

Now we turn to study on geometric solutions to the equation $K=c(c \neq 0)$ in $\mathbf{R}^{3} \times S^{2}$.

Recall that an immersion $f: N \rightarrow \mathbf{R}^{3} \times S^{2}$,

$$
f(u, v)=\left(x_{1}(u, v), x_{2}(u, v), x_{3}(u, v), y_{1}(u, v), y_{2}(u, v), y_{3}(u, v)\right),
$$

is called a geometric solution to $K=c$ if $f$ satisfies the conditions $f^{*} \theta=0, f^{*} \omega=0$ and of course $y_{1}^{2}+y_{2}^{2}+y_{3}^{2}=1$, where

$$
\begin{aligned}
\theta= & y_{1} d x_{1}+y_{2} d x_{2}+y_{3} d x_{3} \\
\omega= & c\left(y_{1} d x_{2} \wedge d x_{3}+y_{2} d x_{3} \wedge d x_{1}+y_{3} d x_{1} \wedge d x_{2}\right) \\
& \quad-\left(y_{1} d y_{2} \wedge d y_{3}+y_{2} d y_{3} \wedge d y_{1}+y_{3} d y_{1} \wedge d y_{2}\right) .
\end{aligned}
$$

First we remark that the Euclidean group $G$ on the Euclidean space $\mathbf{R}^{3}$ acts also on the unit tangent bundle $\mathbf{R}^{3} \times S^{2}$ and $\mathbf{R} \times S^{2}$ transitively such that $\pi_{1}: \mathbf{R}^{3} \times S^{2} \rightarrow \mathbf{R}^{3}$ and $\pi_{2}: \mathbf{R}^{3} \times S^{2} \rightarrow$ $\mathbf{R} \times S^{2}$ are both $G$-equivariant. Moreover the Monge-Ampère system associated to the equation $K=c$ is also $G$-invariant. For each $\left(x_{0}, y_{0}\right) \in \mathbf{R}^{3} \times S^{2}$, the quotient mapping $\pi: G \rightarrow \mathbf{R}^{3} \times S^{2}$, $\pi(g)=g \cdot\left(x_{0}, y_{0}\right)$, is a $C^{\infty}$ fibration. Then there exists a local $C^{\infty}$ section $S=S\left(x_{0}, y_{0} ; \cdot, \cdot\right): V \rightarrow G$ over a neighborhood $V$ of $\left(x_{0}, y_{0}\right)$. Note that $S\left(x_{0}, y_{0}, x, y\right) \in G$ transforms $\left(x_{0}, y_{0}\right)$ to $(x, y)$ for any $(x, y) \in V$.

Let $f:\left(N, x_{0}\right) \rightarrow \mathbf{R}^{3} \times S^{2}$ be a germ of a geometric solution to $K=c$. Take a system of coordinates $(u, v)$ centered at $x_{0}$ of $N$, and fix a $g_{0} \in G$ transforming $f\left(x_{0}\right)$ to $b=(0,0,0 ; 1,0,0) \in$ $\mathbf{R}^{3} \times S^{2}$. Then, for each $\left(u_{0}, v_{0}\right)$ near $(0,0)$, we define $\bar{f}_{u_{0}, v_{0}}:\left(\mathbf{R}^{2},(0,0)\right) \rightarrow\left(\mathbf{R}^{3} \times S^{2}, b\right)$ by

$$
\bar{f}_{u_{0}, v_{0}}(u, v)=g_{0} \cdot S\left(f(0,0) ; f\left(u_{0}, v_{0}\right)\right)^{-1} \cdot f\left(u+u_{0}, v+v_{0}\right) .
$$

Thus, by the homogeneity of the equation $K=c$, we may suppose that $f\left(x_{0}\right)=b=(0,0,0 ; 1,0,0)$. 
Set $p=-y_{2} / y_{1}, q=-y_{3} / y_{1}$. Then we have

$$
\omega=y_{1}\left\{c\left(d x_{2} \wedge d x_{3}-p d x_{3} \wedge d x_{1}-q d x_{1} \wedge d x_{2}\right)-y_{1}^{2} d p \wedge d q\right\}
$$

and $y_{1}^{2}=\frac{1}{1+p^{2}+q^{2}}$. Then the Monge-Ampère system for $K=c$ is locally given by

$$
\left\{\begin{array}{l}
c\left(d x_{2} \wedge d x_{3}-p d x_{3} \wedge d x_{1}-q d x_{1} \wedge d x_{2}\right)-\frac{1}{1+p^{2}+q^{2}} d p \wedge d q=0, \\
d x_{1}-p d x_{2}-q d x_{3}=0 .
\end{array}\right.
$$

Setting $x_{1}=z, x_{2}=x, x_{3}=y$, we have

$$
\left\{\begin{array}{l}
c\left(1+p^{2}+q^{2}\right)^{2} d x \wedge d y-d p \wedge d q=0 \\
d z-p d x-q d y=0
\end{array}\right.
$$

Then, for some local coordinates on $\mathbf{R}^{3}$ and $\mathbf{R} \times S^{2}, \pi_{1}$ and $\pi_{2}$ are given by $\pi_{1}(x, y, z, p, q)=(x, y, z)$ and $\pi_{2}(x, y, z, p, q)=(x p+y q-z, p, q)$ respectively.

As in the case Hess $=c$, we may suppose the mapping $(u, v) \mapsto(x(u, v), q(u, v))$ is a local diffeomorphism. So we assume $x=u$ and $q=v$.

Now, we consider the partial Legendre transformation

$$
L(x, y, z, p, q)=(x, q, z-y q, p,-y)
$$

and its inverse

$$
L^{-1}(x, y, z, p, q)=(x,-q, z-y q, p, y)
$$

(cf. Remark 3.4). Then $L \circ f:\left(\mathbf{R}^{2}, 0\right) \rightarrow\left(\mathbf{R}^{5}, 0\right)$,

$$
L \circ f(u, v)=(u, v, Z, P, Q)
$$

satisfies the equation

$$
\left\{\begin{array}{l}
c\left(1+P^{2}+v^{2}\right)^{2} d Q \wedge d u-d P \wedge d v=0 \\
d Z-P d u-Q d v=0
\end{array}\right.
$$

Note that

$$
Z(u, v)=z \circ f(u, v)-(y \circ f(u, v)) v, P(u, v)=p \circ f(u, v), Q(u, v)=-y \circ f(u, v) .
$$

Then we have

$$
c\left(1+P^{2}+v^{2}\right)^{2} Q_{v}+P_{u}=0, P=Z_{u}, Q=Z_{v},
$$

so we have the Monge-Ampère equation

$$
Z_{u u}+c\left(1+Z_{u}^{2}+v^{2}\right)^{2} Z_{v v}=0
$$

on a function $Z=Z(u, v)$ with $Z(0,0)=0, Z_{u}(0,0)=0, Z_{v}(0,0)=0$. Thus we reduce the problem on geometrical solutions (with projection singularities), via a Legendrian transformation, to the problem on classical solutions to another Monge-Ampère equation.

We compute the "prolongations" of the equation $\left(^{*}\right)$ to obtain the Taylor expansion of $Z$. We have, by setting $(u, v)=(0,0)$,

$$
Z_{u u}(0,0)+c Z_{v v}(0,0)=0
$$

By differentiating by $u$ (resp. by $v$ ) of both sides of $\left(^{*}\right)$ and by setting $(u, v)=(0,0)$, we have

$$
Z_{u u u}(0,0)+c Z_{u v v}(0,0)=0, \quad Z_{u u v}(0,0)+c Z_{v v v}(0,0)=0
$$


By differentiating by $u$ or $v$ of both sides of $(*)$ twice and by setting $(u, v)=(0,0)$, we have

$$
\begin{aligned}
Z_{\text {uuuu }}(0,0)+4 c Z_{u u}(0,0)^{2} Z_{v v}(0,0)+c Z_{u u v v}(0,0) & =0 \\
Z_{u u u v}(0,0)+4 c Z_{u u}(0,0) Z_{u v}(0,0) Z_{v v}(0,0)+c Z_{u v v}(0,0) & =0 \\
Z_{u u v v}(0,0)+4 c\left(Z_{u v}(0,0)^{2}+1\right) Z_{v v}(0,0)+c Z_{v v v v}(0,0) & =0
\end{aligned}
$$

If we set

$$
\begin{array}{r}
Z(u, v)=\frac{1}{2} A u^{2}+B u v+\frac{1}{2} C v^{2}+\frac{1}{6} D u^{3}+\frac{1}{2} E u^{2} v+\frac{1}{2} F u v^{2}+\frac{1}{6} G v^{3} \\
+\frac{1}{24} H u^{4}+\frac{1}{6} I u^{3} v+\frac{1}{4} J u^{2} v^{2}+\frac{1}{6} K v^{3}+\frac{1}{24} L v^{4}+\cdots
\end{array}
$$

then we have

$$
\begin{gathered}
A+c C=0, D+c F=0, E+c G=0 \\
H+4 c A^{2} C+c J=0, I+4 c A B C+c K=0, J+4 c\left(B^{2}+1\right) C+c L=0
\end{gathered}
$$

Therefore we have

$$
\begin{gathered}
A=-c C, D=-c F, E=-c G, \\
I=4 c^{2} B C^{2}-c K, J=-4 c\left(B^{2}+1\right) C-c L, H=-4 c^{3} C^{3}+4 c^{2}\left(B^{2}+1\right) C+c^{2} L .
\end{gathered}
$$

Thus we have

$$
\begin{aligned}
Z(u, v)=- & \frac{c}{2} C u^{2}+B u v+\frac{1}{2} C v^{2}-\frac{c}{6} F u^{3}-\frac{c}{2} G u^{2} v+\frac{1}{2} F u v^{2}+\frac{1}{6} G v^{3} \\
+ & \frac{c^{2}}{24}\left(-4 c C^{3}+4\left(B^{2}+1\right) C+L\right) u^{4}+\frac{c}{6}\left(4 c B C^{2}-K\right) u^{3} v \\
& -\frac{c}{4}\left(4 B^{2} C+4 C+L\right) u^{2} v^{2}+\frac{1}{6} K v^{3}+\frac{1}{24} L v^{4}+\cdots .
\end{aligned}
$$

Note that the original $f$ is given by

$$
x=u, y=Z_{v}, z=Z-Z_{v} v, p=Z_{u}, q=v .
$$

The above procedure gives us all formal solutions to the equation $\left(^{*}\right)$. In fact, the famous theorem of Cauchy-Kovalevskaya ([Jh]) says that the formal solution $Z(u, v)$ is uniquely determined when the initial conditions $Z(0, v)$ and $Z_{u}(0, v)$ are given. In fact, the coefficients of the Taylor expansion of $Z$ up to degree $r$ is determined as explicit polynomials by those of $Z(0, v)$ and $Z_{u}(0, v)$ up to degree $r$. Moreover, if the initial data $Z(0, v)$ and $Z_{u}(0, v)$ are an analytic function, then the solution $Z$ should be an analytic function.

Let $Z(u, v)$ be a $C^{\infty}$ solution to $\left(^{*}\right)$ corresponding to a germ of a geometric solution $f$ to $K=c$. By taking Taylor polynomials of $Z(0, v)$ and $Z_{u}(0, v)$ of arbitrarily high degree, as initial conditions, we get an approximation $\tilde{Z}(u, v)$ of $Z(u, v)$ in $C^{\infty}$ topology, which is an analytic solution to $\left(^{*}\right)$.

By considering $\bar{f}_{u_{0}, v_{0}}:\left(\mathbf{R}^{2}, 0\right) \rightarrow\left(\mathbf{R}^{3} \times S^{2}, b\right)$ defined as above and by considering the Taylor expansion of the $Z$-component of $L \circ \bar{f}_{u_{0}, v_{0}}$, we get a $C^{\infty}$ map-germ $\Phi(f):\left(N, x_{0}\right) \rightarrow \mathbf{R}^{6}$,

$$
\left(u_{0}, v_{0}\right) \mapsto\left(B\left(u_{0}, v_{0}\right), C\left(u_{0}, v_{0}\right), F\left(u_{0}, v_{0}\right), G\left(u_{0}, v_{0}\right), K\left(u_{0}, v_{0}\right), L\left(u_{0}, v_{0}\right)\right)
$$

Because we can control the coefficients $B, C, F, G, K, L$ freely in the approximation precess, we can perturb $f$ into $\tilde{f}$ such that $\Phi(\tilde{f})$ is transverse to a given stratification (or a locally finite family of submanifolds) of $\mathbf{R}^{6}$.

Now consider a stratification of $\mathbf{R}^{6}$ with coordinates $(B, C, F, G, K, L)$ :

$$
W^{0}=\{C \neq 0\}, W^{1}=\{C=0, F \neq 0, G \neq 0\}, W_{1}^{2}=\{C=0, F \neq 0, G=0, L \neq 0\},
$$




$$
\begin{gathered}
W_{2}^{2}=\{C=0, F=0, G \neq 0, L \neq 0\}, W_{1}^{3}=\{C=0, F=0, G=0, L \neq 0\}, \\
W_{2}^{3}=\{C=0, F=0, G \neq 0, L=0\}, W_{3}^{3}=\{C=0, F \neq 0, G=0, L=0\}, \\
W^{4}=\{C=0, F=0, G=0, L=0\} .
\end{gathered}
$$

Note that $W_{1}^{3}, W_{2}^{3}, W_{3}^{3}$ are of codimension 3 and $W^{4}$ is of codimension 4 .

Suppose $\Phi(\tilde{f})$ is transverse to the stratification, namely, transverse to every $W_{j}^{i}$ in $\mathbf{R}^{6}$. The transversality condition implies that the image of $\Phi(\tilde{f})$ does not touch the subset $W_{1}^{3} \cup W_{2}^{3} \cup W_{3}^{3} \cup$ $W^{4}$, so that it is contained in $W^{0} \cup W^{1} \cup W_{1}^{2} \cup W_{2}^{2}$. Then the $u v$-plane is divided into

(i) $C \neq 0, \quad$ (ii) $C=0, F \neq 0, G \neq 0$,

(iii) $C=0, F=0, G \neq 0, L \neq 0, \quad$ (iv) $C=0, F \neq 0, G=0, L \neq 0$.

Now the singular locus of $\pi_{1} \circ f$ is given by

$$
Q_{v}=Z_{v v}=C+F u+G v+\frac{1}{2} J u^{2}+K u v+\frac{1}{2} L v^{2}+\cdots=0 .
$$

We apply Proposition 2.1 to $\pi_{1} \circ f$ at $(0,0)$. If $C \neq 0$, then $\pi_{1} \circ f$ is an immersion at $(0,0)$. If $C=0$, then $\pi_{1} \circ f$ is singular at $(0,0)$. In the case $C=0, \pi_{1} \circ f$ is non-degenerate at $(0,0)$ if and only if $(F, G) \neq(0,0)$. Let $\gamma(t)=(u(t), v(t))$ parametrize the singular locus of $\pi_{1} \circ f$ with $\gamma(0)=(0,0)$. Then we have $F u^{\prime}(0)+G v^{\prime}(0)=0$. We set $\eta(t)=(0,1)$. Then we have $\operatorname{det}\left(\gamma^{\prime}(t), \eta(t)\right)=u^{\prime}(t)$. Suppose $G \neq 0$. Then $u^{\prime}(0) \neq 0$. Hence $\pi_{1} \circ f$ is a cuspidal edge at $(0,0)$. If $C=0, G=0$, then we see $u^{\prime}(0)=0$. Moreover, if $u^{\prime}(0)=0$, then $u^{\prime \prime}(0) \neq 0$ provided $L \neq 0$. Therefore, if $C=0, G=0, F \neq 0, L \neq 0$, then we see $\pi_{1} \circ f$ is a swallowtail at $(0,0)$.

Similarly we apply Proposition 2.1 to $\pi_{2} \circ f$. The singular locus of $\pi_{2} \circ f$ is defined by

$$
P_{u}=Z_{u u}=A+D u+E v+\frac{1}{2} H u^{2}+I u v+\frac{1}{2} J v^{2}+\cdots=0,
$$

while $\eta(t)=(1,0)$. Now recall that $c \neq 0$. Then we see that, if $C \neq 0$, then $\pi_{2} \circ f$ is an immersion at $(0,0)$. If $C=0, G \neq 0, \pi_{2} \circ f$ is a cuspidal edge at $(0,0)$. If $C=0, F=0, G \neq 0, L \neq 0$, then $\pi_{2} \circ f$ is a swallowtail at $(0,0)$.

Therefore in the case (i), both $\pi_{1} \circ f$ and $\pi_{2} \circ f$ are immersions. In the case (ii), both $\pi_{1} \circ f$ and $\pi_{2} \circ f$ are cuspidal edges. In the case (iii), $\pi_{1} \circ f$ is a swallowtail and $\pi_{2} \circ f$ is a cuspidal edge. In the case (iv), $\pi_{1} \circ f$ is a cuspidal edge and $\pi_{2} \circ f$ is a swallowtail.

Thus we have Theorem 1.1 for $K= \pm 1$.

Remark 4.1 For the case $K=c>0$, the equation

$$
Z_{u u}+c\left(1+z_{u}^{2}+v^{2}\right)^{2} Z_{v v}=0
$$

is elliptic. Therefore, by Bernstein's theorem, any solution is analytic ([B1][B2][Ho][Pe]). Hence the method of Cauchy-Kovalevskaya provides all geometric solutions.

In the hyperbolic case $K=c<0$, we can describe all $C^{\infty}$ geometric solutions to $K=c(c<0)$ locally as follows.

Set

$$
\omega=c\left(1+p^{2}+q^{2}\right)^{2} d x \wedge d y-d p \wedge d q, \quad \theta=d z-p d x-q d y .
$$

Then we have two decompositions

$$
\begin{aligned}
& -\omega+\sqrt{-c} d \theta=\left(\sqrt{-c}\left(1+p^{2}+q^{2}\right) d x+d q\right) \wedge\left(\sqrt{-c}\left(1+p^{2}+q^{2}\right) d y-d p\right), \\
& -\omega-\sqrt{-c} d \theta=\left(\sqrt{-c}\left(1+p^{2}+q^{2}\right) d x-d q\right) \wedge\left(\sqrt{-c}\left(1+p^{2}+q^{2}\right) d y+d p\right) .
\end{aligned}
$$

(These decompositions are also used in the proof of Lemma 5.3). 
Assume $f:\left(\mathbf{R}^{2}, 0\right) \rightarrow\left(\mathbf{R}^{5}, 0\right)$ is a geometric solution to $K=c(c<0)$. Moreover we assume $f^{*} d x(0)$ and $f^{*} d q(0)$ are linearly independent. Then we have

$$
\begin{aligned}
& f^{*}\left(\sqrt{-c}\left(1+p^{2}+q^{2}\right) d x+d q\right) \wedge f^{*}\left(\sqrt{-c}\left(1+p^{2}+q^{2}\right) d y-d p\right)=0, \\
& f^{*}\left(\sqrt{-c}\left(1+p^{2}+q^{2}\right) d x-d q\right) \wedge f^{*}\left(\sqrt{-c}\left(1+p^{2}+q^{2}\right) d y+d p\right)=0,
\end{aligned}
$$

$f^{*}\left(\sqrt{-c}\left(1+p^{2}+q^{2}\right) d x+d q\right)(0) \neq 0$ and $f^{*}\left(\sqrt{-c}\left(1+p^{2}+q^{2}\right) d x-d q\right) \neq 0$. Thus we have two foliation $\mathcal{F}, \mathcal{F}^{\prime}$ on the $u v$-plane defined by the equation $f^{*}\left(\sqrt{-c}\left(1+p^{2}+q^{2}\right) d x \pm d q\right)=0$. Each leaf of $\mathcal{F}$ is an integral curve to the differential system $D$ :

$$
\left\{\begin{array}{l}
\sqrt{-c}\left(1+p^{2}+q^{2}\right) d x+d q=0 \\
\sqrt{-c}\left(1+p^{2}+q^{2}\right) d y-d p=0 \\
d z-p d x-q d y=0
\end{array}\right.
$$

and each leaf of $\mathcal{F}^{\prime}$ is an integral curve to the diferential system $D^{\prime}$ :

$$
\left\{\begin{array}{l}
\sqrt{-c}\left(1+p^{2}+q^{2}\right) d x-d q=0 \\
\sqrt{-c}\left(1+p^{2}+q^{2}\right) d y+d p=0 \\
d z-p d x-q d y=0
\end{array}\right.
$$

on the $x y z p q$-space. Thus the geometric solution $f$ is generated by a one-parameter family of integral curves to $D$ (resp. $D^{\prime}$ ). The differential systems $D$ and $D^{\prime}$ are called the Monge characteristic systems. Compare our conclusion with the classical Monge-Ampère-Goursat theorem stating that any classical solution is obtained by a one-parameter family of integral curves to the Monge characteristic system $D\left(\right.$ resp. $\left.D^{\prime}\right)([\mathrm{Go}]$ Ch.2, [Mat $]$ ). Note that the differential system $D \subset T \mathbf{R}^{5}$ (resp. $D^{\prime} \subset T \mathbf{R}^{5}$ ) has the growth vector $(2,3,5)$, namely, $D$ is of constant rank 2, $D^{(2)}=D+[D, D]$ is of constant rank 3 and $D^{(3)}=D^{(2)}+\left[D, D^{(2)}\right]=T \mathbf{R}^{5}$ (cf. [Mon]). Two foliations $\left(\mathcal{F}, \mathcal{F}^{\prime}\right)$ on the uv-plane form the Chebyshev net on the image of $\pi_{1} \circ f$ in $\mathbf{R}^{3}$, which consists of asymptotic lines. Note that $\pi_{1} \circ f$ restricted to each characteristic curve is an immersion, and therefore each asymptotic line is an immersed curve beyond the singular locus. Of course, the angle $\psi$ (the Chebyshev angle) of asymptotic lines tends to 0 or $\pi$ on the singular locus.

Then $f$ is described, via a Bäcklund transformation, by a classical solution $\psi=\psi(t, s)$ to the sine-Gordon equation $\psi_{t s}=-c \sin \psi$ (cf. [P-T] Ch.3). Here $t$ (resp. $s$ ) is the arclength coordinate of the characteristic curves of $D$ (resp, $D^{\prime}$ ). Note that the system of coordinates $(t, s)$ on $\mathbf{R}^{2}$ may be different from the system of coordinates $(u, v)$ satisfying $x=u, q=v$. Then, as classically well-known, the solution $\psi$ is determined by two initial data $\alpha(t)=\psi(t, 0), \beta(s)=\psi(0, s)$ with $\alpha(0)=\beta(0)$ ([Go][Mat]). Thus we have an alternative method for the construction of transversal approximations of geometric solutions to $K=c(c<0)$. For the recent progress on the initial value problem of sine-Gordon equations and the representation formula for surfaces with $K=c(c<0)$ related to integrable systems, see [To1][To2] for instance.

\section{Singularities of developable surfaces.}

First we show Theorem 1.6. We will show that, for a generic geometric solution

$$
f(u, v)=(x(u, v), y(u, v), z(u, v), p(u, v), q(u, v))
$$

to Hess $=0$ (or equivalently, $K=0$ ), $\pi \circ f$ has as singularities only cuspidal edges and swallowtails.

First note that $\pi_{2} \circ f$ is of rank $\leq 1$. Thus we see $\pi_{1} \circ f$ is of rank $\geq 1$. Suppose $\pi_{1} \circ f$ is singular at a point $\left(u_{0}, v_{0}\right)$. Then $\pi_{1} \circ f$ is of rank 1 at $\left(u_{0}, v_{0}\right)$, so that $\pi_{2} \circ f$ is of rank 1 at $\left(u_{0}, v_{0}\right)$ as well. Then, up to equivalence at each such a point, we may suppose

$$
f(u, v)=(u, y(u, v), z(u, v), p(u, v), v) .
$$


Though we can show Theorem 1.6 for $K=0$ by the same calculations as in the previous section by setting $c=0$, here we will give its proof in a rather direct manner.

From $d z=p d x+q d y=p d u+v d y$ and $d p \wedge d q=d p \wedge d v=0$, we have

$$
p_{v}+y_{u}=0, \quad p_{u}=0 .
$$

From the condition $p_{u}=0$, we set

$$
p=p(v)=A+B v+\frac{1}{2} C v^{2}+\frac{1}{6} D v^{3}+\cdots .
$$

Then, from the condition $y_{u}=-p_{v}$, we set

$$
y(u, v)=-B u-C u v-\frac{1}{2} D u v^{2}-\cdots+\tilde{A}+\tilde{B} v+\frac{1}{2} \tilde{C} v^{2}+\frac{1}{6} \tilde{D} v^{3}+\cdots .
$$

Then we have

$$
z(u, v)=\tilde{\tilde{A}}+A u-\frac{1}{2} C u v^{2}-\frac{1}{3} D u v^{3}-\cdots+\frac{1}{2} \tilde{B} v^{2}+\frac{1}{3} \tilde{C} v^{3}+\frac{1}{6} \tilde{D} v^{4}+\cdots
$$

and

$$
\tilde{z}=p x+q y-z=-\tilde{\tilde{A}}+\tilde{A} v+\frac{1}{2} \tilde{B} v^{2}+\frac{1}{6} \tilde{C} v^{3}+\cdots .
$$

The singular locus of $\pi_{1} \circ f$ is given by

$$
y_{v}=-C u-D u v-\cdots+\tilde{B}+\tilde{C} v+\frac{1}{2} \tilde{D} v^{2}+\cdots=0 .
$$

If $\tilde{B} \neq 0$ we see $\pi \circ f$ is immersive at $(u, v)=(0,0)$. Suppose $\tilde{B}=0$. Then $f$ is non-degenerate if and only if $(C, \tilde{C}) \neq(0,0)$. Let $\gamma(t)=(u(t), v(t))$ parametrize the singular locus. Set $\eta(t)=(0,1)$. Then $\operatorname{det}\left(\gamma^{\prime}(t), \eta(t)\right)=u^{\prime}(t)$. If $\tilde{B}=0, \tilde{C} \neq 0, C \neq 0$, then $\operatorname{det}\left(\gamma^{\prime}(0), \eta(0)\right)=u^{\prime}(0) \neq 0$. Then, by Proposition 2.1, we see $\pi_{1} \circ f$ is a cuspidal edge. If $\tilde{B}=0, \tilde{C}=0, C \neq 0$, then we have $u^{\prime}(0)=0$. Since $u^{\prime \prime}(0)=(\tilde{D} / 2 C) v^{\prime}(0)^{2}$, we see $u^{\prime \prime}(0) \neq 0$ if and only if $\tilde{D} \neq 0$.

As seen above, the geometric solution $f$ is determined by two functions $p(u, v)=p(v)$ and $y(u, v)+p_{v}(v) u$ of one variable $v$. From a pair of two functions $(\varphi(v), \psi(v))$ we get a geometric solution

$$
f(u, v)=\left(u, \psi(v)-\varphi_{v}(v) u, \int\left(\varphi-v \varphi_{v}\right) d u+v\left(\psi_{v}-u \varphi_{v v}\right) d v, \varphi(v), v\right)
$$

to Hess $=0$. We define a mapping from a neighborhood of $\left(u_{0}, v_{0}\right)$ to $\mathbf{R}^{4}$ by

$$
(u, v) \mapsto(\tilde{B}, \tilde{C}, C, \tilde{D})=\left(\psi_{v}(v)-\varphi_{v v}(v) u, \psi_{v v}(v)-\varphi_{v v v}(v) u, \varphi_{v v}(v), \psi_{v v v}(v)-\varphi_{v v v v}(v) u\right)
$$

For a generic $(\varphi(v), \psi(v))$, we have that $\varphi_{v v}$ vanishes at a finite number of points, where $\psi_{v}(v) \neq 0$. Therefore $(\tilde{B}, C) \neq(0,0)$. Moreover two vectors

$$
\left(\psi_{v}(v), \psi_{v v}(v), \psi_{v v v}(v)\right) \text { and }\left(\varphi_{v v}(v), \varphi_{v v v}(v), \varphi_{v v v v}(v)\right)
$$

are linearly independent for any $v$. Then, if $\tilde{B}=\psi_{v}(v)-\varphi_{v v}(v) u=0$ for some $(u, v)$, then $(\tilde{C}, \tilde{D})=\left(\psi_{v v}(v)-\varphi_{v v v}(v) u, \psi_{v v v}(v)-\varphi_{v v v v}(v) u\right) \neq(0,0)$.

Thus only the following cases occur on the $u v$-plane for a generic geometric solution:
(i) $\tilde{B} \neq 0$,
(ii) $\tilde{B}=0, \tilde{C} \neq 0, C \neq 0$,
(iii) $\tilde{B}=0, \tilde{C}=0, C \neq 0, \tilde{D} \neq 0$.

In the case (i), $\pi \circ f$ is an immersion, in the case (ii), $\pi_{1} \circ f$ is a cuspidal edge, and, in the case (iii), $\pi_{1} \circ f$ is a swallowtail. Thus we have Theorem 1.6.

To show Proposition 1.7, we recall Lemma 11 of [I-Mo]: 
Lemma 5.1 Let $f:\left(\mathbf{R}^{2}, 0\right) \rightarrow M$ be an open umbrella and $H:(M, f(0)) \rightarrow(\mathbf{R}, 0)$ a smooth function-germ. Suppose $d H(f(0)) \neq 0$ and $H \circ f=0$. Then the kernel of $d H(f(0)): T_{f(0)} M \rightarrow \mathbf{R}$ coincides with the contact hyperplane in $T_{f(0)} M$.

An integral mapping $f:\left(\mathbf{R}^{2}, 0\right) \rightarrow M$ is called full if it has the property in Lemma 5.1. An integral mapping $f$ is called formally full if, for any formal complex valued function $\hat{H}$ at $f(0)$ in $M$, the conditions $\hat{H} \circ \hat{f}_{\mathbf{C}}=0$ and $d \hat{H}(0) \neq 0$ imply that the kernel of $d \hat{H}(f(0))$ : $T_{f(0)} M \otimes \mathbf{C} \rightarrow \mathbf{C}$ coincides with the complexification of the contact hyperplane in $T_{f(0)} M$. Here $\hat{f}_{\mathbf{C}}$ is the complexification of the formal Taylor series of $f$ at 0 in $\mathbf{R}^{2}$. If $f$ is full, then it is formally full.

For our treatment including the elliptic cases $(c>0)$, we need a slightly strict result than Lemma 5.1:

Lemma 5.2 An open umbrella $f:\left(\mathbf{R}^{2}, 0\right) \rightarrow M$ is formally full of rank one.

Proof: By the contact invariance of the assumption and conclusion, we may suppose

$$
f=\left(u, v^{2}, u v^{3}, v^{3}, \frac{3}{2} u v\right):\left(\mathbf{R}^{2}, 0\right) \rightarrow\left(\mathbf{R}^{5}, 0\right)
$$

with respect to the contact form $\theta=d z-p d x-q d y$. Set

$$
\hat{H}(x, y, z, p, q)=A x+B y+C z+D p+E q+F(x, y, z, p, q)
$$

where $A, B, C, D, E$ are complex numbers and $F$ is a formal power series starting from second order terms. The assumption means that $A, B, C, D, E$ are not all zero, and that

$$
A u+B v^{2}+C u v^{3}+D v^{3}+E \frac{3}{2} u v+F\left(u, v^{2}, u v^{3}, v^{3}, \frac{3}{2} u v\right)=0,
$$

as a formal power series of $u$ and $v$. Then we see $A=0, B=0, D=0, E=0$. Thus the kernel of $d \hat{H}(0)$ coincides with $\{\theta=d z=0\}$ at 0 in $\mathbf{R}^{5}$.

Then Proposition 1.7 follows from the following:

Lemma 5.3 Let $f:\left(\mathbf{R}^{2}, 0\right) \longrightarrow M$ be an integral map-germ. Suppose $f$ is a generalized geometric solution to Hess $=c, c \neq 0$, (resp. $K=c, c \neq 0)$, of rank $\geq 1$. Then $f$ is never formally full.

Proof: First we consider the case Hess $=c, c \neq 0$. By the assumption we have $f^{*} \theta=0, f^{*} \omega=0$, where $\theta=d z-p d x-q d y, \omega=c d x \wedge d y-d p \wedge d q$. Now consider the complex valued 2-forms

$$
\begin{aligned}
(\sqrt{-c} d x+d q) \wedge(\sqrt{-c} d y-d p)= & -c d x \wedge d y+d p \wedge d q+\sqrt{-c}(d p \wedge d x+d q \wedge d y) \\
= & -\omega+\sqrt{-c} d \theta
\end{aligned}
$$

and

$$
\begin{aligned}
(\sqrt{-c} d x-d q) \wedge(\sqrt{-c} d y+d p)= & -c d x \wedge d y+d p \wedge d q-\sqrt{-c}(d p \wedge d x+d q \wedge d y) \\
= & -\omega-\sqrt{-c} d \theta
\end{aligned}
$$

Then we see $f^{*}(\sqrt{-c} d x+d q) \wedge f^{*}(\sqrt{-c} d y-d p)=0$ and $f^{*}(\sqrt{-c} d x-d q) \wedge f^{*}(\sqrt{-c} d y+d p)=0$, near 0 on $\mathbf{R}^{2}$. On the other hand, since $f$ is of rank $\geq 1$, we see

$$
\left(f^{*} d x\right)(0),\left(f^{*} d y\right)(0),\left(f^{*} d p\right)(0),\left(f^{*} d q\right)(0)
$$

are not all zero. Therefore

$$
f^{*}(\sqrt{-c} d x+d q), f^{*}(\sqrt{-c} d y-d p), f^{*}(\sqrt{-c} d x-d q), f^{*}(\sqrt{-c} d y+d p)
$$


are not all zero at 0 in $\mathbf{R}^{2}$. Suppose, for instance, $f^{*}(\sqrt{-c} d x+d q)(0) \neq 0$. Then, regarding $\sqrt{-c} x+q$ as a formal power series with complex coefficients, we have $(\sqrt{-c} x+q) \circ \hat{f}_{\mathbf{C}}=a u+b v+\cdots$, with $(a, b) \neq(0,0)$. Since $f^{*}(\sqrt{-c} d x+d q) \wedge f^{*}(\sqrt{-c} d y-d p)=0$, we see there exists a formal power series $\hat{H}_{1}$ of $\sqrt{-c} x+q, \sqrt{-c} y-p$ satisfying $\hat{H}_{1}\left((\sqrt{-c} x+q) \circ \hat{f}_{\mathbf{C}},(\sqrt{-c} y-p) \circ \hat{f}_{\mathbf{C}}\right)=0$ and $d \hat{H}(0) \neq 0$. Then we may set $\hat{H}(x, y, z, p, q)=\hat{H}_{1}(x+\sqrt{-c} q, y-\sqrt{-c} p)$. Therefore $f$ is not formally full.

In the case $K=c, c \neq 0$, as seen in the previous section, we have $f^{*} \theta=0, f^{*} \omega=0$, where

$$
\theta=d z-p d x-q d y, \quad \omega=c\left(1+p^{2}+q^{2}\right)^{2} d x \wedge d y-d p \wedge d q .
$$

Since also in this case we have similar decompositions into two ways

$$
\left(\sqrt{-c}\left(1+p^{2}+q^{2}\right) d x+d q\right) \wedge\left(\sqrt{-c}\left(1+p^{2}+q^{2}\right) d y-d p\right)=-\omega+\sqrt{-c} d \theta
$$

and

$$
\left(\sqrt{-c}\left(1+p^{2}+q^{2}\right) d x-d q\right) \wedge\left(\sqrt{-c}\left(1+p^{2}+q^{2}\right) d y+d p\right)=-\omega-\sqrt{-c} d \theta
$$

we have a formal complex valued function $\hat{H}(x, y, z, p, q)$ with $\hat{H} \circ \hat{f}_{\mathbf{C}}=0, d \hat{H}(0) \neq 0,\{d \hat{H}(0)=$ $0\} \neq D_{f(0)}$, the complexified contact hyperplane in $T_{f(0)} M \otimes \mathbf{C}$. Therefore $f$ is not formally full.

Remark 5.4 In general, for a hyperbolic Monge-Ampère system $\{\theta, \omega\}$ on $\mathbf{R}^{5}$, there exist independent 1-forms $\omega_{1}, \omega_{2}, \omega_{3}, \omega_{4}$ such that $\omega \equiv \omega_{1} \wedge \omega_{2} \equiv \omega_{3} \wedge \omega_{4}$ mod.d $d \theta$. Even in an elliptic case, we have similar decompositions into formal 1-forms in the complex category. See for instance [I-L][B-G-H]. Thus the same proof as in Lemma 5.3 works to conclude the assertion: If an integral map-germ $f:\left(\mathbf{R}^{2}, 0\right) \rightarrow\left(\mathbf{R}^{5}, 0\right)$ is formally full and of rank 1 , then $f$ can not be a generalized geometric solution to any hyperbolic nor any elliptic Monge-Ampère system on $\mathbf{R}^{5}$.

\section{References}

[A-G-V] V.I. Arnol'd, S.M. Gusein-Zade, A.N. Varchenko, Singularities of Differentiable Maps I, Birkhäuser, 1985.

[B1] S.N. Bernstein, Sur la nature analytique des solutions des équations aux dérivées partielles du second ordre, Math. Ann., 59 (1904), 20-76.

[B2] S.N. Bernstein, Über ein geometrisches Theorem und seine Anwendung auf die partiellen Differentialgleichungen von elliptischen Typus, Math.Z., 26(1927), 551-558.

[B-G-H] R.Bryant, P.Griffiths and L.Hsu, Toward a geometry of differential equations, in Geometry, Topology and Physics for Raul Bott, ed. by S.-T.Yau, International Press (1994), pp.1-76.

[B-H] R.L. Bryant, L. Hsu, Rigidity of integral curves of rank 2 distributions, Invent. math., 114 (1993), $435-461$.

[C] E. Calabi, Improper affine hyperspheres of convex type and a generalization of a theorem by K. Jörgens, Michigan Math. J., 5 (1958), 105-126.

[F-M-M] L. Ferrer, A. Martínez, F. Milán, An extension of a theorem by K. Jörgens and a maximum principle at infinity for parabolic affine spheres, Math. Z., 230 (1999), 471-486.

[G-M-M] J.A. Gálvez, A. Martínez, F. Milán, Flat surfaces in hyperbolic 3-space, Math. Ann., 316 (2000), 419-435.

[G-G] M. Golubitsky, V. Guillemin, Stable Mappings and Their Singularities, Graduate Texts in Math., 14, Springer-Verlag, New York (1973).

[Go] E. Goursat, Leçons sur l'intégrations des équations aux dérivées partielles du second ordre: I, Hermann, Paris, (1926).

[Hi] D. Hilbert, Ueber Flähen von constanter Gaussscher Krümmung, Trans. Amer. Math. Soc., 2-1 (1901), 87-99. 
[Ho] E. Hopf, Über den funktionalen, insbesondere den analytischen Character der Lösungen elliptischer Differentialbleichungen zweiter Ordnung, Math. Z., 34-2 (1931), 191-233.

[Is] G. Ishikawa, Singularities of developable surfaces, in London Mathematical Society Lecture Notes Series, 263 (1999), pp. 403-418.

[I-Ma] G. Ishikawa and Y. Machida, Monge-Ampère systems with Lagrangian pairs, in preparation.

[I-Mo] G. Ishikawa and T. Morimoto, Solution surfaces of the Monge-Ampère equation, Differential Geometry and its Applications, 14 (2001), 113-124.

[I-L] T.A. Ivey and J.M. Landsberg, Cartan for beginners: differential geometry via moving frames and exterior differential systems, Amer. Math. Soc., (2003).

[Iz] S. Izumiya, Generating families of developable surfaces in $\mathbf{R}^{3}$, Preprint.

[Jh] F. John, Partial Differential Equations, 4th. ed., Springer-Verlag, New York (1982).

[Jr] K. Jörgens, Über die Lösungen der Differentialgleichung $r t-s^{2}=1$, Math. Ann., 127 (1954), $130-134$.

[Kob] Shimpei Kobayashi, A talk in the singularity seminar at Hokkaido University, 2003.

[K-R-S-U-Y] M. Kokubu, W. Rossman, K. Saji, M. Umehara, K. Yamada, Singularities of flat fronts in hyperbolic 3-space, to appear in Pacific J. Math..

[K-U-Y] M. Kokubu, M. Umehara, K. Yamada, Flat fronts in hyperbolic 3-space, arXiv: math.DG/0301224.

[L] V.V. Lychagin, Contact geometry and non-linear second-order differential equations, Uspekhi Mat. Nauk, 34 (1979), 137-165. English transl. Russsian Math. Surveys 34 (1979), 149-180.

[L-R-C] V.V. Lychagin, V.N. Rubtsov, I.V. Chekalov, A classification of Monge-Ampère equations, Ann. Sci. Èc. Norm. Sup., 26 (1993), 281-308.

[M-M] Y. Machida and T. Morimoto, On decomposable Monge-Ampère equations, Lobachevskii J. Math. 3 (1999), 185-196.

[Mar] A. Martínez, Improper affine maps, to appear in Math. Z..

[Mat] M. Matsuda, On the Monge-Ampère equations, (in Japanese), Sûgaku, 24-2 (1972), 100-118.

[Mc] R. McLachlan, A gallery of constant-negative-curvature surfaces, Math. Intelligencer, 16-4 (1994), $31-37$.

[M1] T. Morimoto, La géométrie des équations de Monge-Ampère, C. R. Acad. Sci. Paris, 289 (1979), 25 -28 .

[M2] T. Morimoto, Monge-Ampère equations viewed from contact geometry, in Banach Center Publications vol. 39 "Symplectic Singularities and Geometry of Gauge Fields" , (1998), pp. 105-120.

[Mon] R. Montgomery, A Tour of Subriemannian Geometry, Their Geodesics and Applications, Mathematical Surveys and Monographs, vol. 91, Amer. Math. Soc., (2002).

[N] J.C.C. Nitsche, Elementary proof of Bernstein's theorem on minimal surfaces, Ann. of Math. 66-3 (1957), 543-544.

[N-S] K. Nomizu and T. Sasaki, Affine differential geometry, Cambridge Tracts in Math., 111, Cambridge Univ. Press (1994).

[O] B. O'Neill, Elementary Differential Geometry, Academic Press (1966).

[P-T] R.S. Palais, C.-l. Terng, Critical Point Theory and Submanifold Geometry, Lecture Notes in Math., 1353, Springer-Verlag, (1988).

[Pe] I.G. Petrovskii, Lectures on Partial Differential Equations, Interscience Publishers, (1954).

[Po] A.V. Pogorelov, On the improper convex affine hypersurfaces, Geometriae Dedicata, 1 (1972), 33-46.

[To1] Magdalena Toda, Weierstrass-type representation of weakly regular pseudospherical surfaces in Euclidean space, Balkan J. Geom. Appl., 7-2 (2002), 87-136.

[To2] Magdalena Toda, Initial value problems of the sine-Gordon equation and geometric solutions, arXiv: math.DG/0307270. 
[Ts] M. Tsuji, Hyperbolic equations, waves and the singularity theory, Functional-analytic and complex methods, their interactions, and applications to partial differential equations (Graz, 2001), World Sci. Publishing (2001), pp. 3-22.

[Z] V. M. Zakalyukin, Lagrangian and Legendrian singularities, Functional Anal. Appl., 10 (1976), 23-31.

\section{Go-o ISHIKAWA}

Department of Mathematics, Hokkaido University, Sapporo 060-0810, JAPAN.

E-mail : ishikawa@math.sci.hokudai.ac.jp

Yoshinori MACHIDA

Numazu College of Technology, 3600 Ooka, Numazu-shi, Shizuoka, 410-8501, JAPAN.

E-mail : machida@numazu-ct.ac.jp 